\title{
HYPERFINE INTERACTION IN Gd IN DIAMAGNETIC, METALLIC HOSTS
}

\author{
R. B. FRANKEL \\ Physik Department der Technischen Hochschule, München, Germany \\ and \\ Francis Bitter National Magnet Laboratory $\ddagger$. Massachusetts Institute of Technology. \\ Cambridge, Massachusetts, USA
}

\begin{abstract}
Mossbauer effect data for dilute. paramagnetic $\mathrm{Gd}$ in the diamagnetic, metallic hosts $\mathrm{YAl}_{2}, \mathrm{YbAl}_{2}$ and $\mathrm{Al}$ are analysed and yield a local conduction electron polarization contribution to the Gd hyperfine field of $+140 \mathrm{kOe}$.
\end{abstract}

Hyperfine fields at Gd nuclei in metallic. magnetically ordered materials may be considered to have three main sources [1-3]: (1) core polarization; (2) local conduction electron polar ization; (3) neighbor effects, including conduction electron polarization, overlap effects and covalency. The magnitude of contribution (1) may be obtained from measurements of $\mathrm{Gd}^{3+}$ hyperfine interactions in diamagnetic insulators $(-340 \mathrm{kOe})$. The magnitude of contribution (2) could be obtained from measurements of $\mathrm{Gd}^{3+}$ hyperfine interactions in metallic, diamagnetic hosts, assuming that (1) has the same magnitude in metals as in insulators. In this letter. recent measurements by Persson et al. [4] are analysed to obtain the magnetic field and temperature dependence of the paramagnetic hyperfine structure [5] in $\mathrm{Gd}^{3}+$ in the cubic, diamagnetic, metallic hosts $\mathrm{YAl}_{2}, \mathrm{YbAl}_{2}$ and $\mathrm{Al}$. and hence contribution (2) in these materials.

Persson et al. [4] measured the splitting between the $\Delta m=+1$ and -1 component of the $2+\stackrel{\mathrm{E}_{2}}{\rightarrow} 0^{+} 89 \mathrm{keV}$ transition in $156 \mathrm{Gd}$, for Gd met al and $\mathrm{GdFe}_{2}$ in longitudinal, external magnetic fields at $4.2^{\circ} \mathrm{K}$. The sources were also in the external field at low temperature and the observed splitting is the vector difference of the splitting in the absorber and source.

$$
\Delta E=2 g_{2+\mu_{\mathrm{N}}}\left(\boldsymbol{H}_{\mathrm{NS}}-\boldsymbol{H}_{\mathrm{NA}}\right),
$$

where $\Delta E$ is the observed splitting, $\boldsymbol{H}_{\mathrm{NS}}$ is the field at the nucleus in the source and $\boldsymbol{H}_{\mathrm{NA}}$ is the

\footnotetext{
$\$$ Supported by the US Air Force Office of Scientific Research.
}

field at the nucleus in the absorber. The meas ured splitting gives $\boldsymbol{H}_{\mathrm{NS}}$ because [5]

$$
\boldsymbol{H}_{\mathrm{NA}}=\boldsymbol{H}_{\mathrm{hfA}} \pm \boldsymbol{H}_{\mathrm{O}},
$$

where $\boldsymbol{H}_{\mathrm{hfA}}$ is the hyperfine splitting in the absorber and $H_{\mathrm{O}}$ is the external field. $\mathrm{Gd}^{3+}$ has a $4 \mathrm{f}^{7}$ configuration and an ${ }^{8} \mathrm{~S}_{\frac{7}{2}}$ ground state; hence we expect the paramagnetic hyperfine field in the source to have a Brillouin function dependence on $H_{\mathrm{O}} / T$, i.e.,

$$
\begin{aligned}
\boldsymbol{H}_{\mathrm{hfS}} & =\boldsymbol{H}_{\mathrm{NS}}-\boldsymbol{H}_{\mathrm{o}}^{\mathrm{S}} \\
& \left.=\boldsymbol{H}_{\mathrm{hf}}^{\mathrm{S}} B_{\frac{1}{2}} \frac{/ g \mu \mathrm{B} H_{\mathrm{o}}^{\mathrm{S}}}{k T}\right)
\end{aligned}
$$

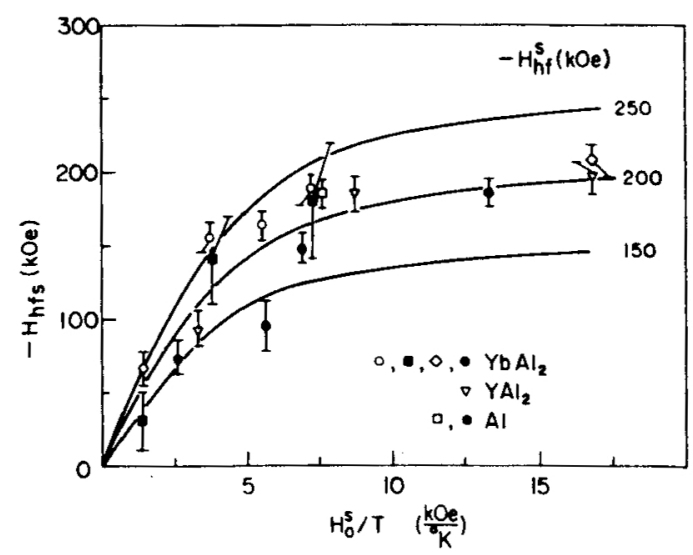

Fig. 1. Hyperfine field at the Gd nucleus plotted as a function of the external field divided by temperature. The solid curves are theoretical (eq. (3)) for three values of the saturation hyperfine field. The data are taken from ref. 4 , except $\otimes$ taken from ref. 5 . 
where $\boldsymbol{H}_{\mathrm{hf}}^{\mathbf{S}}$ is the saturation hyperfine field and $\boldsymbol{H}_{\mathrm{O}}^{\mathrm{S}}$ is the external field at the source. We take the electronic $g$ factor $=2$; small $g$-shifts will not affect the determination of $\boldsymbol{H}_{\mathrm{hf}}^{\mathrm{S}}$. In fig. 1 , $H_{\text {hfS }}$ from the data of Persson [4] are plotted as a function of $H_{0} \mathrm{~S} / T$. The solid curves are calculated from eq. (3), for three values of $\boldsymbol{H}_{\mathrm{hf}}^{\mathrm{S}}$. The data for the three hosts follow the Brillouin function behavior reasonably well, and indicate a saturation hyperfine field $H_{\text {hf }}^{S}=-200 \pm 25 \mathrm{kOe}$. Using $-340 \mathrm{kOe}$ as the core polarization contribution, this gives $+140 \mathrm{kOe}$ as the local conduction electron contribution.

In Gd metal, $H_{\mathrm{hf}}=-300 \mathrm{kOe}[4]$ and all three contributions to the hyperfine field are present. Hüfner [1] and Zmora et al. [3] estimated contribution (2) to be $+240 \mathrm{kOe}$, i.e., almost twice as large as in $\mathrm{YbAl}_{2}, \mathrm{YAl}_{2}$ and $\mathrm{Al}$. This change is consistent with a larger change density at the $\mathrm{Gd}$ nucleus in $\mathrm{Gd}$ metal compared with $\mathrm{Gd}$ in the dilute alloys [6], because the magnitude of (2) depends on the 's'-like electron density of states at the Fermi level.

I wish to thank W. Henning, A. Misetich and R. L. Mössbauer for useful discussions.

\section{References}

1. S. Hüfner, Phys. Rev. Letters 19 (1967) 1034

2. A.J. Freeman and R. E. Watson in: Treatise on magnetism Vol. IIA, eds. Suhl and Rado (Academic Press, N. Y., 1965)

3. H. Zmora, M. Blau and S. Ofer, Phys. Letters 28A (1969) 668 .

4. B. Persson, H. Blumberg and M. Bent, Phys, Letters $27 \mathrm{~A}(1968) 189$

5. H. Maletta, R. B. Frankel, W. Henning and R. L. Mössbauer, Phys. Letters 28A (1969) 557.

6. Quoted by K. E. Rehm, W. Henning and P. Kienle, Phys. Rev. Letters 22 (1969) 790. 